\title{
Pembrolizumab given concomitantly with chemoradiation and as maintenance therapy for locally advanced head and neck squamous cell carcinoma: KEYNOTE-412
}

\author{
Jean-Pascal Machiels*,1 (iD), Yungan Tao², Barbara Burtness ${ }^{3}$, Makoto Tahara4, Lisa Licitra ${ }^{5}$, \\ Danny Rischin ${ }^{6}$, John Waldron ${ }^{7}$, Christian Simon $^{8}$, Vincent Gregoire ${ }^{9}$, Kevin Harrington ${ }^{10}$, \\ Gustavo Vasconcelos Alves ${ }^{11}$, lane Pinto Figueiredo Lima ${ }^{12}$, Yoann Pointreau ${ }^{13}$, Brett G M \\ Hughes $^{14}$, Sercan Aksoy ${ }^{15}$, Marcin Hetnal ${ }^{16}$, Joy $Y \mathrm{Ge}^{17}$, Holly Brown ${ }^{17}$, Jonathan Cheng ${ }^{17}$, \\ Behzad Bidadi ${ }^{17}$ \& Lillian L Siu ${ }^{18}$ \\ ${ }^{1}$ Department of Medical Oncology, Institut Roi Albert II, Cliniques Universitaires Saint-Luc \& Institut de Recherche Clinique et \\ Expérimentale, Université Catholique de Louvain (UCLouvain), Avenue Hippocrate 10, Brussels, Belgium \\ ${ }^{2}$ Department of Radiation Oncology, Gustave Roussy Cancer Campus, 114 Rue Edouard Vaillant, 94800, Villejuif, France \\ ${ }^{3}$ Department of Medicine, Yale University School of Medicine \& Yale Cancer Center, 333 Cedar Street, New Haven, CT 06510, USA \\ ${ }^{4}$ Department of Head \& Neck Medical Oncology, National Cancer Center Hospital East, 6-Chome-5-1 Kashiwanoha, Kashiwa, \\ Chiba, 27-8577, Japan \\ ${ }^{5}$ Department of Oncology, Fondazione IRCCS Istituto Nazionale dei Tumori and University of Milan, Via Giacomo Venezian 1 , \\ 20133, Milan, Italy \\ ${ }^{6}$ Department of Medical Oncology, Peter MacCallum Cancer Centre, 305 Grattan Street, Melbourne, VIC, 3000, Australia \\ ${ }^{7}$ Department of Radiation Oncology University of Toronto, Princess Margaret Cancer Centre, 610 University Avenue, Toronto, ON, \\ M5G 2M9, Canada \\ ${ }^{8}$ Department of Otorhinolaryngology, University Hospital CHUV, Rue du Bugnon 21, 1011, Lausanne, Switzerland \\ ${ }^{9}$ Department of Radiation Oncology, Centre Leon Berard, 28 Prom. Lea et Napoleon Bullukian, 69008, Lyon, France \\ ${ }^{10}$ Division of Radiotherapy \& Imaging, The Institute of Cancer Research, London, SW3 6JB, UK \\ ${ }^{11}$ Centro Integardo de Pesquisa em Oncologia, Hospital Nossa Senhora da Conceição, Avenida Francisco Trein, 596 - Cristo \\ Redentor Porto Alegre - RS, 91350-200, Brazil \\ ${ }^{12}$ Crio Centro Regional Integrado de Oncologia, R. Francisco Calaca, 1300 - Alvaro Weyne, Fortaleza - CE, 60335-480, Brazil \\ ${ }^{13}$ Department of Radiation Oncology, Institut Inter-Regional de Cancerologie-Centre Jean Bernard/Clinique Victor Hugo, 72000 , \\ Le Mans, France \\ ${ }^{14}$ Cancer Care Services, Royal Brisbane \& Women's Hospital, Butterfield Street, Herston QLD 4029, Australia \& School of Medicine, \\ University of Queensland, 20 Weightman Street, Herston, QLD, 4006, Australia \\ ${ }^{15}$ Hacettepe University, Cancer Institute, Department of Medical Oncology, 06100, Ankara, Turkey \\ ${ }^{16}$ Faculty of Medicine \& Health Sciences, Andrzej Frycz Modrzewski Krakow University, Amethyst Radiotherapy Centre, Rydygier \\ Hospital, Krakow, Poland, Ztotej Jesieni 1, Krakow, Poland \\ ${ }^{17}$ Merck \& Co., Inc., 2000 Galloping Hill Road, Kenilworth, NJ 07033, USA \\ ${ }^{18}$ Division of Medical Oncology \& Hematology, Princess Margaret Cancer Centre, University Health Network, 610 University \\ Avenue, Toronto, ON, M5G 2M9, Canada \\ *Author for correspondence: Tel.: +322 764 5457; Fax: +322 764 5428; jean-pascal.machiels@uclouvain.be
}

Current treatment guidelines for patients with locally advanced head and neck squamous cell carcinoma (HNSCC) recommend multimodal treatment, including chemoradiation therapy (CRT) or surgery followed by radiation, with or without chemotherapy. The immune checkpoint inhibitor pembrolizumab has previously demonstrated antitumor activity in recurrent and/or metastatic HNSCC in large Phase III trials. For patients with locally advanced disease, Phase $\mathrm{Ib}$ data on the use of pembrolizumab in combination with chemoradiation have shown the approach to be safe and feasible. We describe here the design and rationale for KEYNOTE-412, a randomized, double-blind, Phase III trial investigating pembrolizumab or placebo administered concurrently with CRT and as maintenance treatment in patients with locally advanced HNSCC.

Clinical Trial Registration: NCT03040999 (ClinicalTrials.gov)

First draft submitted: 2 March 2020; Accepted for publication: 17 April 2020; Published online: 3 June 2020 
Keywords: chemoradiation $\bullet$ head and neck cancer $\bullet$ immune checkpoint blockade $\bullet$ PD-1 • PD-L1 • pembrolizumab

Head and neck cancer, the sixth most common cancer worldwide [1], is diagnosed in approximately 706,000 new patients annually and accounts for 358,000 deaths every year [2]. Among these patients, $60 \%$ have locally or regionally advanced disease at the time of diagnosis [3]. Head and neck squamous cell carcinoma (HNSCC) constitutes more than $90 \%$ of head and neck cancers and most commonly affects the oral cavity, oropharynx, hypopharynx and larynx [1]. The global incidence of HNSCC varies based on environmental and genetic risk factors and on persistent infection with the oncogenic human papillomavirus (HPV), primarily HPV-16 [1,4,5]. Overall incidence is influenced by tobacco use and alcohol consumption in patients with HPV-negative disease and HPV-driven oropharyngeal cancer [1]. In fact, HPV-driven HNSCC has led to a $>25 \%$ increase in HNSCC incidence in USA during the past decade [1]; it is anticipated that the incidence will continue to rise.

Treatment for newly diagnosed HNSCC is determined by an experienced multidisciplinary team after an assessment based on the location of the primary tumor, the stage of the disease, and the expected functional outcomes [3]. For patients with early-stage disease, single-modality treatment with surgery or radiation is generally recommended. For patients with locally advanced (LA) HNSCC, the standard of care is chemoradiation therapy (CRT) or surgery followed by radiation, with or without chemotherapy [6]. The 5-year overall survival (OS) rate for patients with HPV-negative stage III/IV HNSCC remains low at 40-50\%, but patients with HPV-associated oropharyngeal cancer have a better prognosis $[4,7]$. The prognosis for patients with stage 3 (T4 or N3, TNM Classification of Malignant Tumours, 8th edition) HPV-associated HNSCC is similar to that for patients with HPV-negative HNSCC (5-year OS is 50\%) $[7,8]$.

Evidence is mounting that radiation can act as an immunologic stimulus, potentially counteracting the immunosuppressive tumor microenvironment and providing a strong rationale for the combination of radiation and immunotherapeutic agents [9]. PD-1 is an immune checkpoint receptor known to play a role in tumor immune evasion, and its ligand, PD-L1, is also highly expressed in HNSCC tumors on the tumor cells and on the components of the tumor microenvironment [10-12]. The anti-PD-1 agents pembrolizumab and nivolumab have been approved by the US FDA as monotherapy for the treatment of patients with recurrent and/or metastatic (R/M) HNSCC with disease progression on or after platinum-containing chemotherapy [13,14]. Pembrolizumab has also recently been approved for first-line treatment of patients with R/M HNSCC, as monotherapy for those patients whose tumors express PD-L1 (combined positive score $\geq 1$ ) and in combination with platinum and fluorouracil regardless of PD-L1 expression [13]. While the safety of these agents in patients with LA HNSCC has been established in early-phase studies, more data are needed to demonstrate superior efficacy to CRT in this patient population.

Recently, results were reported from a preplanned interim analysis of the Phase III JAVELIN Head and Neck 100 study of the combination of the anti-PD-L1 agent avelumab and CRT versus CRT alone in previously untreated LA HNSCC. The JAVELIN Head and Neck 100 study was terminated based on the recommendation of the independent data monitoring committee showing that avelumab plus CRT versus CRT alone is unlikely to show significant improvement in the primary end point of progression-free survival [15]. Given that pembrolizumab is an anti-PD-1 agent, whereas avelumab is an anti-PD-L1 agent and the efficacy of pembrolizumab has been established in patients with R/M HNSCC, the results of the JAVELIN Head and Neck 100 study are not expected to influence the conduct of KEYNOTE-412 (ClinicalTrials.gov: NCT03040999).

\section{KEYNOTE-412 trial}

KEYNOTE-412 (NCT03040999) is a randomized, double-blind, Phase III study evaluating the efficacy and safety of pembrolizumab or placebo given concomitantly with CRT followed by maintenance pembrolizumab therapy or placebo in patients with LA HNSCC.

\section{Background \& rationale}

Pembrolizumab is a potent and highly selective humanized monoclonal antibody of the immunoglobulin G4- $\kappa$ isotype designed to block the interaction between PD-1 and its ligands, PD-L1 and PD-L2 [13]. Pembrolizumab has demonstrated robust antitumor activity and a favorable safety profile in multiple tumor types and is currently approved in more than 80 countries for one or more advanced malignancies, including previously untreated $\mathrm{R} / \mathrm{M}$ HNSCC or R/M HNSCC with disease progression on or after platinum-containing chemotherapy [13]. The durability of response and tolerability of pembrolizumab monotherapy in patients with R/M HNSCC has been demonstrated in early-phase trials, including the Phase Ib KEYNOTE-012 study with long-term follow-up [16], 
and in patients previously treated with platinum and cetuximab in the Phase II KEYNOTE-055 study [17]. The Phase III KEYNOTE-040 study evaluated pembrolizumab monotherapy in patients whose HNSCC progressed after platinum therapy [18], whereas the Phase III KEYNOTE-048 study evaluated pembrolizumab, alone or in combination with chemotherapy, in patients who did not previously receive systemic therapy for R/M disease [19].

In the Phase III KEYNOTE-040 study (NCT02252042), 495 patients with HNSCC that progressed during or after platinum-containing treatment for R/M disease or whose disease recurred or progressed within 3-6 months of previous platinum-containing therapy for LA disease were randomly assigned to receive pembrolizumab monotherapy or investigator's choice of standard of care (SOC; methotrexate, docetaxel, or cetuximab) [18]. After a median follow-up of 7.5 months in the pembrolizumab arm and 7.1 months in the SOC arm, a clinically meaningful increase in median OS was observed in patients receiving pembrolizumab compared with SOC (hazard ratio [HR], 0.80; 95\% confidence interval $[\mathrm{CI}], 0.65-0.98$; nominal $\mathrm{p}=0.0161$ ). Treatment-related adverse events (AEs) were reported in $63 \%$ of patients treated with pembrolizumab compared with $84 \%$ of patients treated with SOC. Overall, the pembrolizumab safety profile was better than that of SOC and consistent with the safety profile of pembrolizumab in previous studies; no new toxicities were observed [18].

The KEYNOTE-048 study (NCT02358031) enrolled 882 patients who had not previously received systemic therapy for R/M HNSCC and who were randomly assigned to receive pembrolizumab monotherapy, pembrolizumab plus chemotherapy (cisplatin or carboplatin plus 5-FU), or cetuximab plus chemotherapy (the EXTREME regimen) [19]. At the final analysis, OS with pembrolizumab monotherapy was found to be noninferior to the EXTREME regimen in the total population (HR: 0.83; 95\% CI: 0.70-0.99). OS with pembrolizumab monotherapy was superior to EXTREME in patients with tumor PD-L1 combined positive score (CPS) of $\geq 20$ (HR: 0.58; 95\% CI: 0.44-0.78) and in patients with tumor PD-L1 CPS of $\geq 1$ (HR: 0.74; 95\% CI: 0.61-0.90). Pembrolizumab plus chemotherapy significantly improved OS compared with EXTREME in the total population (HR: 0.72 ; 95\% CI: 0.60-0.87). OS with pembrolizumab plus chemotherapy was superior to EXTREME in patients with tumor PD-L1 CPS of $\geq 20$ (HR: 0.60; 95\% CI: 0.45-0.82) and in patients with tumor PD-L1 CPS of $\geq 1$ (HR: 0.65 ; 95\% CI: $0.53-0.80$ ). Responses were durable and the safety profile was favorable in both pembrolizumab arms [19]. Results from both of these large Phase III trials support pembrolizumab as monotherapy and as part of combination therapy in the treatment of relapsed or metastatic disease.

Additionally, a single-arm, open-label, Phase Ib trial investigated the safety and tolerability of pembrolizumab in combination with standard cisplatin-based, definitive CRT in patients with stage III-IVB HNSCC (NCT02586207). Patients were treated with pembrolizumab at a fixed dose of $200 \mathrm{mg}$ intravenously approximately 7 days before the initiation of CRT and every 3 weeks during CRT (two concomitant doses) with five additional doses following CRT. CRT consisted of weekly cisplatin $40 \mathrm{mg} / \mathrm{m}^{2}$ intravenously for six doses $\left(240 \mathrm{mg} / \mathrm{m}^{2}\right.$ maximum) given concurrently with radiation at a dose of 2 Gy once daily for 35 fractions (total 70 Gy). Coprimary end points included safety and complete response rate at treatment end. A safety analysis was performed in 27 patients with oropharyngeal, hypopharyngeal, and laryngeal LA HNSCC of any HPV status; 21 patients completed all planned doses of pembrolizumab, 27 completed the full radiation dose, and 23 received the target dose of cisplatin. Six patients discontinued treatment because of immune-related AEs $(n=3$; grade 2 peripheral motor neuropathy; grade 3 aspartate aminotransferase elevation; grade 1 Lhermitte-like syndrome) or for protocol reasons $(n=3$; early neck dissection $[n=2]$; prolonged hospitalization $[n=1]$ ); one patient died of concurrent illness unrelated to treatment [20]. Combination therapy was deemed safe and did not significantly limit radiation or chemotherapy dosing. The study is ongoing with $34 \mathrm{HPV}$-positive and $23 \mathrm{HPV}$-negative patients enrolled in the expansion cohorts to evaluate antitumor activity [20]. Results from a recent analysis of the efficacy and safety in the HPV-positive expansion cohort showed that most patients (79\%) had disease of intermediate risk. Approximately $85 \%$ of patients (29/34) experienced complete response based on imaging and/or surgery and $97.1 \%$ (95\% CI: 80.9-99.6) demonstrated progression-free survival at 1 year. Additional observations revealed no impact on CRT delivery and no new safety signals [21].

Support for the hypothesis of pembrolizumab treatment for LA HNSCC is demonstrated by the antitumor activity of pembrolizumab monotherapy for R/M HNSCC, in conjunction with the initial safety results of pembrolizumab plus CRT in patients with LA disease. The addition of pembrolizumab to standard CRT may also address the need for an effective treatment option for patients with LA HNSCC, with the potential to translate into superior effectiveness and improved patient outcomes. 


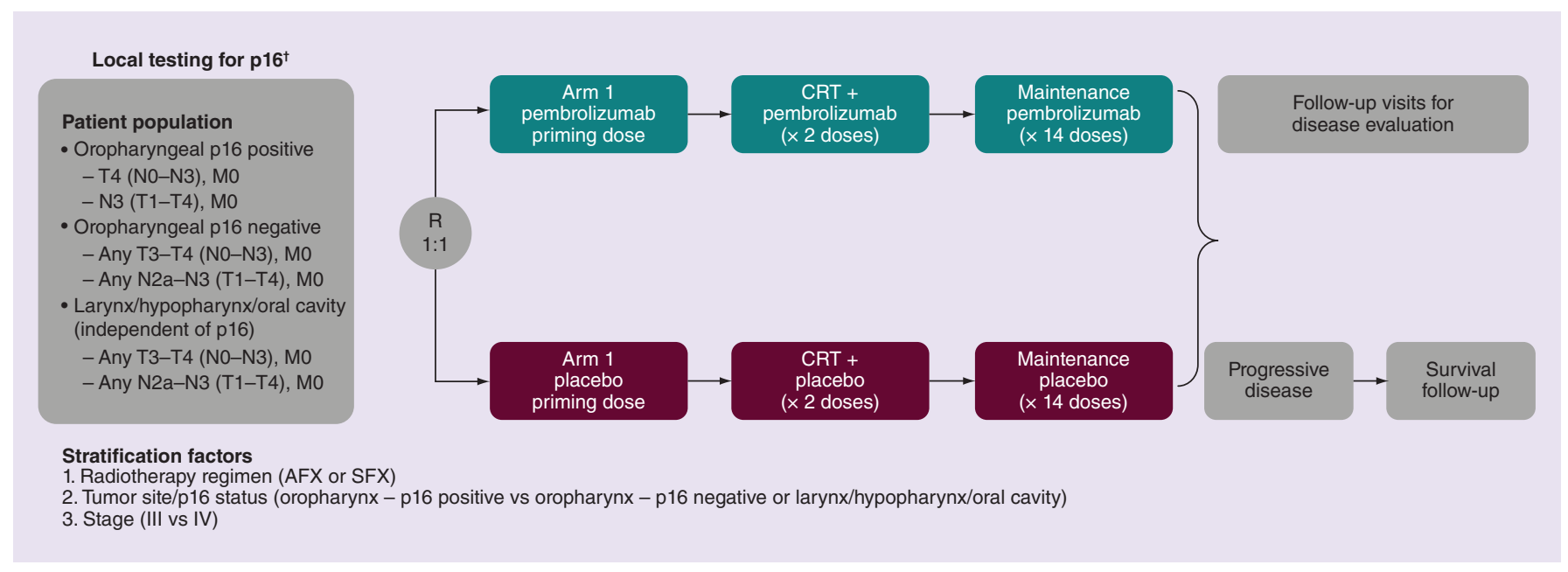

Figure 1. KEYNOTE-412 study design.

†Expression of p16 is highly correlated with human papillomavirus in oropharyngeal cancer. Tumors outside the oropharynx will be considered human papillomavirus negative regardless of results of p16 staining. Tumors were staged according to the 7 th edition of the American Joint Committee on Cancer staging manual for patients enrolled before 1 January 2018, and according to the 8th edition of the American Joint Committee on Cancer staging manual for patients enrolled on or after that date $[8,23]$.

AFX: Accelerated fractionation; CRT: Chemoradiation therapy; R: Randomization; SFX: Standard fractionation.

\section{Study design \& treatment}

KEYNOTE-412 (NCT03040999) is a randomized, double-blind, placebo-controlled, Phase III study of intravenous (iv.) infusion of pembrolizumab $200 \mathrm{mg}$ or placebo every 3 weeks (Q3W) in combination with CRT and as maintenance therapy for a total of 17 doses (Figure 1). This dosing schedule and duration was selected in order to evaluate the efficacy of pembrolizumab given in combination with curative intent therapy with the primary end point of event-free survival (see the Study End points section). In addition, 1 year of adjuvant pembrolizumab has been shown to be effective in improving outcomes and providing clinically meaningful benefit to patients with melanoma. In the KEYNOTE-054 study, 1 year of adjuvant pembrolizumab treatment versus placebo resulted in significantly longer recurrence-free survival in patients with high-risk stage III melanoma [22].

This study occurred at 151 sites in 21 countries. Cisplatin $100 \mathrm{mg} / \mathrm{m}^{2}$ IV Q3W will be given concurrently with radiotherapy in both study arms. The radiotherapy regimen consisted of accelerated fractionation (AFX) or standard fractionation (SFX). For patients treated with AFX RT, 70 Gy was administered in a total of 35 fractions over 6 weeks at a frequency of six fractions per week. SFX 70 Gy will be administered for a total of 35 fractions over 7 weeks at a frequency of five fractions per week. Treatment will continue until centrally confirmed disease progression, unacceptable toxicity, intercurrent illness that prevents further administration of treatment, investigator's decision to withdraw the patient, nonadherence to treatment or trial procedures, administrative reasons requiring cessation of treatment, or the patient has received 17 administrations of pembrolizumab/placebo ( $\sim 1$ year).

The trial is split into three treatment phases. The first phase includes the pembrolizumab/placebo priming dose, followed by CRT in combination with two additional pembrolizumab/placebo doses given every 3 weeks (duration, 8 weeks). The second phase includes pembrolizumab/placebo maintenance dosing (14 doses) during post-CRT follow-up (total treatment duration, $\sim 1$ year). The third phase includes posttreatment follow-up.

Eligible patients were randomly assigned 1:1 to pembrolizumab or placebo using an interactive voice/integrated web response system. Randomization was stratified according to radiotherapy regimen (AFX vs SFX), tumor site, p16 status (oropharynx - p16 positive vs oropharynx - p16 negative or larynx/hypopharynx/oral cavity HNSCC), and tumor stage (III vs IV). Treatment assignments are masked to patients, investigators and sponsor personnel.

\section{Eligibility criteria}

Eligibility criteria are described in Table 1 . In brief, inclusion criteria include patients aged $\geq 18$ years with a pathologically proven new diagnosis of LA HNSCC who were eligible for definitive CRT and were not considered for primary surgery based on investigator decision. Patients with oropharyngeal p16-positive disease were required to have T4 (N0-N3), M0 or N3 (T1-T4), M0 tumors. Patients with oropharyngeal p16-negative disease were required 


\section{Table 1. Eligibility criteria.}

\section{Inclusion criteria}

\section{- Male or female}

- Aged $\geq 18$ years

- Pathologically proven new diagnosis of squamous cell carcinoma Oropharyngeal $\mathrm{p} 16$ positive

- T4 (NO-N3), M0 or

- N3 (T1-T4), M0

- Oropharyngeal p16 negative

- Any T3-T4 (NO-N3), M0 or

- Any N2a-3 (T1-T4), M0

- Larynx/hypopharynx/oral cavity (independent of $\mathrm{p} 16$ )

- Any T3-T4 (NO-N3), M0 or

- Any N2a-3 (T1-T4), M0

- Patients with oral cavity tumors must have unresectable disease

- HPV status for oropharyngeal cancer defined by p16 IHC testing using CINteC (Ventana Medical Systems Inc., AZ, USA) p16 histology assay and a 70\% cut-off point

- Tissue for PD-L1 biomarker analysis from core or excisional biopsy

- Evaluable tumor burden (measurable and/or nonmeasurable tumor lesions) assessed by CT or MRI per RECIST v1.1

- Eligible for definitive CRT and not considered for primary surgery based on investigator decision

- ECOG PS 0 or 1 within 10 days of treatment initiation

- Negative urine or serum pregnancy test result within $72 \mathrm{~h}$ before randomization (women)

- Willing to use an adequate method of contraception for the course of the study through 180 days after the last dose of study medication

- Adequate hematologic function, defined as ANC $\geq 1500 / \mu \mathrm{l}$, platelet count $\geq 100,000 / \mu \mathrm{l}$, and hemoglobin $\geq 9.0 \mathrm{~g} / \mathrm{dl}$ or $\geq 5.6 \mathrm{mmol} / \mathrm{l}$

- Adequate renal function, defined as measured or calculated creatinine clearance $\geq 60 \mathrm{ml} / \mathrm{min}$ and calcium (corrected for albumin) $\leq 11.5 \mathrm{mg} / \mathrm{dl}$ or $\leq 2.9 \mathrm{mmol} / \mathrm{l}$

- Adequate hepatic function, defined as total bilirubin $\leq 1.5 \times$ ULN or direct bilirubin $\leq$ ULN for those with total bilirubin levels $>1.5 \times$ ULN, and ALT/AST levels $\leq 2.5 \times$ ULN

- Adequate coagulation function, defined as INR $\leq 1.5 \times$ ULN unless the patient is receiving anticoagulant therapy as long as PT or aPTT is within the therapeutic range

- Written informed consent

\section{Exclusion criteria}

- Cancer outside the oropharynx, larynx, hypopharynx, or oral cavity, such as nasopharyngeal, sinus, other paranasal, or other unknown primary HNC

- Multiple synchronous tumors

- Previous systemic therapy, targeted therapy, radiotherapy, or radical surgery for HNC

- Grade $\geq 2$ audiometric hearing loss

- Grade $\geq 2$ neuropathy

- Grade 3/4 bleeding from underlying malignancy

- Known history of HIV, HBV, or HCV infection

- Known active central nervous system metastases and/or carcinomatous meningitis

- Active autoimmune disease that has necessitated systemic treatment (other than replacement therapy) in the past 2 years

- Diagnosis of immunodeficiency, receiving systemic steroid therapy or any other form of immunosuppressive therapy within 7 days before the first dose of study treatment, or history of allogeneic tissue or solid organ transplantation

- Active infection necessitating systemic therapy

- Pneumonitis (past or current) necessitating steroid therapy

- History of diagnosed and/or treated hematologic or primary solid tumor malignancy, unless in remission for at least 5 years before randomization

- History or current evidence of any condition, therapy, or laboratory abnormality that might confound the study results or interfere with study participation

- Severe comorbidities that in the investigator's opinion might hamper participation in the study and/or treatment administration

- Known psychiatric or substance abuse disorder that would impede cooperation with study requirements

- Pregnant or breastfeeding, or expecting to conceive within the projected study duration

- Previous treatment with anti-PD-1, anti-PD-L1, or anti-PD-L2 agent or an agent directed to another stimulatory or coinhibitory T-cell receptor (e.g., CTLA4, OX-40, CD137)

- Known history of severe hypersensitivity to any of the study drugs or their analogs

- Immunized with live vaccine within 30 days before the first dose of study treatment

- Participation in a study of an investigational agent or device within 4 weeks before the first dose of study treatment

ANC: Absolute neutrophil count; aPTT: Activated partial thromboplastin time; CRT: Chemoradiation therapy; CT: Computed tomography; ECOG PS: Eastern Cooperative Oncology Group performance status; HBV: Hepatitis B virus; HCV: Hepatitis C virus; HNC: Head and neck cancer; HPV: Human papillomavirus; IHC: Immunohistochemistry; INR: International normalized ratio; MRI: Magnetic resonance imaging; PT: Prothrombin time; RECIST v1.1: Response evaluation criteria in solid tumors version 1.1; ULN: Upper limit of normal.

to have any T3-T4 (N0-N3), M0 or any N2a-3 (T1-T4), M0 tumors. Patients with larynx/hypopharynx/oral cavity disease, independent of p16, will be required to have any T3-4 (N0-N3), M0 or any N2a-3 (T1-T4), M0 tumors. Tumors were staged according to the 7 th edition of the American Joint Committee on Cancer (AJCC) staging manual for patients enrolled before 1 January 2018, and according to the 8th edition of the AJCC staging manual for patients enrolled on or after that date $[8,23]$.

\section{Planned sample size, study period \& statistical analysis}

Target accrual for this trial was approximately 780 patients. The study started in April 2017, and enrollment completed in May 2019. The estimated study completion date is June 2023.

All randomly assigned patients will be included in the efficacy analysis and will be analyzed by randomized treatment group. All randomly assigned patients who received at least one dose of study drug will be assessed in the safety analysis and will be analyzed by treatment received. The treatment effect will be evaluated using a stratified log-rank test. Hazard ratios will be estimated using a stratified Cox regression model. Event rates over time will be estimated using the Kaplan-Meier method. Interim analyses for efficacy and safety are planned, and results will be reviewed by an external data monitoring committee; this committee will also review safety data periodically throughout the study.

\section{Study end points}

The primary end point of the trial is event-free survival (EFS) using Response Evaluation Criteria in Solid Tumors version 1.1 (RECIST v1.1). EFS is defined as the time from the date of randomization to the date of first record 
of progression per RECIST v1.1 by blinded independent central review ([a] locoregional progression or recurrence or [b] distant metastasis), salvage surgery at the primary tumor site when invasive cancer is present, neck dissection performed $>20$ weeks after completion of CRT when invasive cancer is present, or death from any cause. The key secondary end point is OS, which is defined as the time from randomization to death from any cause. Other secondary end points include safety and patient-reported outcomes (PROs); PROs are assessed using the European Organisation for the Research and Treatment of Cancer Quality of Life Questionnaire (EORTC QLQ) core 30 items (C30) and head and neck module (H\&N35) as well as the EuroQoL-5D (EQ-5D). Exploratory end points include potential predictive biomarkers and immune dynamics in the subgroup of patients with oropharyngeal p16-negative or larynx/hypopharynx/oral cavity HNSCC and the overall population.

\section{Study procedures}

At the discretion of the investigator, computed tomography (CT) or magnetic resonance imaging (MRI) will be used to assess tumor response and disease progression; the same method of assessment will be used for each patient. All patients underwent clinical and radiologic (CT or MRI and fluorodeoxyglucose positron emission tomography [FDG-PET] or FDG-PET/CT) assessment within 6 weeks of randomization. Follow-up imaging was acquired at week 12 after CRT and will be repeated every 3 months for 3 years after randomization and then every 6 months for years 4 and 5. At 12 weeks after CRT, an FDG-PET or FDG-PET/CT scan was acquired as part of neck management.

Safety and tolerability will be assessed by clinical review of all relevant parameters, including AEs, laboratory tests, and vital signs. AEs will be graded and recorded throughout the trial and follow-up period using the National Cancer Institute Common Terminology Criteria for Adverse Events version 4.0. Patients will be monitored throughout the study and for 30 days after the end of treatment ( 90 days for serious AEs). Treatment-related toxicity will also be recorded up to 5 years after the end of treatment.

PRO assessments (EORTC QLQ-C30, EORTC-H\&N35 and EQ-5D) will be administered electronically, beginning with a baseline assessment at cycle 1 until year 5; assessments will be administered before drug administration, AE evaluation, and disease status. Tumor PD-L1 expression will be assessed by central evaluation. PD-L1 will be measured using CPS, which is calculated by the number of PD-L1-positive cells (tumor cells, lymphocytes and macrophages) divided by the total number of tumor cells, multiplied by 100 . Exploratory biomarker investigation may include genetic, RNA, and proteomics analyses of blood and tumor samples.

\section{Conclusion}

Despite the currently available treatment options for patients with LA HNSCC, there is a need for better therapies in the curative setting for this patient population. With observed benefit in patients with R/M HNSCC, the immune checkpoint inhibitor pembrolizumab warrants further investigation in LA HNSCC. The Phase Ib study investigating the safety and tolerability of pembrolizumab in combination with CRT in patients with LA HNSCC suggests that this approach may be safe and to date has had no evidence of new safety concerns. Herein we described the methodology for the ongoing Phase III KEYNOTE-412 (NCT03040999) study investigating the safety and efficacy of pembrolizumab versus placebo combined with concurrent CRT and as maintenance therapy for LA HNSCC. Results from the KEYNOTE-412 (NCT03040999) study will elucidate the role of the combination of CRT with pembrolizumab in patients with LA HNSCC, a patient population for whom the 5-year survival rates remain poor at $40-50 \%[4,7]$. In addition, biomarker data from this study may assist in determining factors that influence the efficacy of immunotherapy and in selecting patient subpopulations most likely to benefit from treatment with pembrolizumab.

\footnotetext{
Author contributions

JP Machiels, B Burtness, L Licitra, J Waldron, JY Ge, H Brown, J Cheng, B Bidadi, and LL Siu conceived, designed, or planned the study. Drafting of the manuscript: Y Tao and L Licitra. JP Machiels, Y Tao, B Burtness, M Tahara, L Licitra, J Waldron, C Simon, V Gregoire, Y Pointreau, BGM Hughes, M Hetnal, S Aksoy, JY Ge, H Brown, J Cheng, B Bidadi, and LL Siu critically reviewed or revised the manuscript for important intellectual content. All authors reviewed the final version and are in agreement with the content and approved of the decision to submit.
}

Acknowledgments

The authors thank the patients and their families and caregivers and all investigators and site personnel. 
Background \& rationale

- Current treatment guidelines for patients with locally advanced head and neck squamous cell carcinoma (HNSCC) recommend multimodal treatment, including chemoradiation therapy (CRT) or surgery followed by radiation with or without chemotherapy. Only safety data with immune checkpoint inhibitors in patients with locally advanced HNSCC are available.

- KEYNOTE-412 (ClinicalTrials.gov:NCT03040999) is a randomized, double-blind, Phase III study evaluating the efficacy and safety of pembrolizumab or placebo given concomitantly with CRT followed by maintenance pembrolizumab or placebo therapy in patients with locally advanced HNSCC.

- The antitumor activity and safety of pembrolizumab monotherapy in HNSCC has been established in patients with recurrent and/or metastatic disease in the Phase III KEYNOTE-040 and KEYNOTE-048 studies, as well as for pembrolizumab given concurrently with platinum-based chemotherapy in the KEYNOTE-048 study.

- Interim data from a Phase Ib single-arm trial investigating the safety and tolerability of pembrolizumab in combination with CRT in patients with stage III-IVB HNSCC revealed that the treatment regimen is tolerable and feasible.

- The therapeutic combination of concomitant pembrolizumab and CRT, followed by maintenance pembrolizumab, may be beneficial for patients with locally advanced HNSCC.

Patients and treatment

- Approximately 780 patients with previously untreated, locally advanced HNSCC have been enrolled; efficacy and safety data are awaited.

- Eligible patients were randomly assigned to receive pembrolizumab or placebo in combination with the current standard treatment of CRT (cisplatin plus fractionated radiation).

Primary end point

- The primary end point is event-free survival.

Conclusion

- The KEYNOTE-412 (NCT03040999) trial was designed to evaluate the clinical utility of pembrolizumab in combination with CRT and as maintenance therapy in patients with locally advanced HNSCC.

Financial \& competing interests disclosure

Funding for this research was provided by Merck Sharp \& Dohme Corp., a subsidiary of Merck \& Co., Inc., Kenilworth, NJ, USA. JP Machiels reports advisory board fees from Roche, AstraZeneca, Bayer, Innate, Merck Serono, Boehringer Ingelheim, Bristol-Myers Squibb, Novartis, Janssen, Incyte, Cue Biopharma, ALX Oncology, and Pfizer; travel expenses from Bristol-Myers Squibb, Amgen, and Pfizer; uncompensated advisory board for Merck Sharp \& Dohme; and safety monitoring committee for PsiOxus, Debiopharm, and Nanobiotix. Y Tao reports research funding from Debiopharm, Merck Sharp \& Dohme, and Pfizer; and travel/accommodation expenses from Merck Sharp \& Dohme and Merck Serono. B Burtness reports consulting/advisory fees from Merck Sharp \& Dohme, Celgene, CueBioPharma, Aduro, Glaxo Smith Kline, Genentech, AstraZeneca, Bristol-Myers Squibb, Debiopharm, Macrogenics, and IOBiotech; speaker fees from Physician's Education Resource, CCO Pharma, and OncLive; and travel/gifts/in-kind payments from Merck Sharp \& Dohme and Aduro. M Tahara reports grants and personal fees from Merck Sharp \& Dohme, Bayer, AstraZeneca, Eisai, Bristol-Myers Squibb, Ono Pharmaceutical, Pfizer, Rakuten Medical; personal fees from Merck Serono, Celgene, and Amgen; and grants from Novartis. L Licitra reports research funding to her institution from AstraZeneca, Boehringer Ingelheim, Eisai, Merck Serono, Merck Sharp \& Dohme, Novartis, and Roche; consulting fees from AstraZeneca, Bayer, Bristol-Myers Squibb, Boehringer Ingelheim, Debiopharm, Eisai, Merck Serono, Merck Sharp \& Dohme, Novartis, Roche, and Sobi; speaker fees from AstraZeneca, Bayer, Bristol-Myers Squibb, Boehringer Ingelheim, Debiopharm, Eisai, Merck Serono, Merck Sharpe \& Dohme, Novartis, Roche, and Sobi; advisory board fees from Ipsen, GlaxoSmithKline, Health \& Life SRL, and Doxapharma; personal fees for public speaking/teaching in medical meetings from Immuno-Oncology hubXchange, Incyte Biosciences Italy SRL, Amgen, and Nanobiotix. D Rischin reports research funding from Merck Sharp \& Dohme, Regeneron, GlaxoSmithKline, Sanofi, Roche, and Bristol-Myers Squibb. K Harrington reports research funding to his institution from AstraZeneca, Boehringer Ingelheim, Merck Sharp \& Dohme, and Replimune; and honoraria and advisory/consulting fees from AstraZeneca, Bristol-Myers Squibb, Boehringer Ingelheim, Merck Serono, Merck Sharp \& Dohme, Oncolys BioPharma, Pfizer, and Replimune. G Alves reports research funding from Merck Sharp \& Dohme, Roche, AstraZeneca, Merck Serono, Bristol-Myers Squibb, BeiGene, and Pfizer; and personal fees from Roche, Merck Serono, and Pfizer. BGM Hughes reports consulting/advisory fees from Merck Sharp \& Dohme, BristolMyers Squibb, Roche, AstraZeneca, Pfizer, Eisai, and Boehringer Ingelheim. Y Pointreau: advisory board fees from Merck Serono, Bristol-MyersSquib, Merck Sharp \& Dohme. S Aksoy reports honoraria from Roche, Novartis, Astellas, Pfizer, and Abdi Ibrahim Pharmaceuticals; and advisory board fees from Roche, Novartis, Eli Lilly, Abdi Ibrahim Pharmaceuticals, Bristol-Myers Squibb, and Merck Sharp \& Dohme. JY Ge, J Cheng, and B Bidadi report employment with and stock options from Merck \& Co., Inc. LL 
Siu reports research funding to her institution from Merck Sharp \& Dohme, Novartis, Bristol-Myers Squibb, Pfizer, Boehringer Ingelheim, GlaxoSmithKline, Roche/Genentech, Karyopharm, AstraZeneca/Medimmune, Celgene, Astellas, Bayer, AbbVie, Amgen, Symphogen, Intensity Therapeutics, Mirati Therapeutics, Shattuck Labs, Avid Radiopharmaceuticals; advisory board fees from Merck Sharp \& Dohme, AstraZeneca/Medimmune, Pfizer, Celgene, MorphoSys, Roche/Genentech, Geneseeq Technology, Loxo, Oncorus, Symphogen, Seattle Genetics, GlaxoSmithKline, Voronoi, Treadwell Therapeutics, Arvinas, Tessa Therapeutics, and Navire Pharma; spouse stock ownership in Agios; and spouse leadership at Treadwell Therapeutics. The authors have no other relevant affiliations or financial involvement with any organization or entity with a financial interest in or financial conflict with the subject matter or materials discussed in the manuscript apart from those disclosed.

Medical writing and/or editorial assistance was provided by HC Cappelli and D Mitra of the ApotheCom pembrolizumab team (PA, USA). This assistance was funded by Merck Sharp \& Dohme Corp., a subsidiary of Merck \& Co., Inc., NJ, USA.

Ethical conduct of research

The authors state that they have obtained appropriate institutional review board approval and have followed the principles outlined in the Declaration of Helsinki for all human experimental investigations. In addition, informed consent has been obtained from the participants involved.

Open access

This work is licensed under the Attribution-NonCommercial-NoDerivatives 4.0 Unported License. To view a copy of this license, visit http://creativecommons.org/licenses/by-nc-nd/4.0/

\section{References}

Papers of special note have been highlighted as: $\bullet$ of interest; $\bullet \bullet$ of considerable interest

1. Vigneswaran N, Williams MD. Epidemiologic trends in head and neck cancer and aids in diagnosis. Oral Maxillofac. Surg. Clin. North Am. 26(2), 123-141 (2014).

2. Bray F, Ferlay J, Soerjomataram I, Siegel RL, Torre LA, Jemal A. Global cancer statistics 2018: GLOBOCAN estimates of incidence and mortality worldwide for 36 cancers in 185 countries. CA Cancer J. Clin. 68(6), 394-424 (2018).

3. National Comprehensive Cancer Network. NCCN Clinical Practice Guidelines in Oncology 2019 Head and Neck Cancers v3.2019. Plymouth Meeting, PA: National Comprehensive Cancer Network (2019).

4. Ang KK, Harris J, Wheeler R et al. Human papillomavirus and survival of patients with oropharyngeal cancer. N. Engl. J. Med. 363(1), 24-35 (2010).

5. Gillison ML, Chaturvedi AK, Anderson WF, Fakhry C. Epidemiology of human papillomavirus-positive head and neck squamous cell carcinoma. J. Clin. Oncol. 33(29), 3235-3242 (2015).

6. Gregoire V, Lefebvre JL, Licitra L Felip E. EHNS-ESMO-ESTRO Guidelines Working Group. Squamous cell carcinoma of the head and neck: EHNS-ESMO-ESTRO Clinical Practice Guidelines for diagnosis, treatment and follow-up. Ann. Oncol. 21(Suppl.5), v184-v186 (2010).

7. Bower R, Green VL, Kuvshinova E et al. Maintenance of head and neck tumor on-chip: gateway to personalized treatment? Future Sci. OA 3(2), FSO174 (2017).

8. American Joint Committee on Cancer. TNM Classification of Malignant Tumours. (8th Edition) American Joint Committee on Cancer, IL, USA (2018).

9. Kang J, Demaria S, Formenti S. Current clinical trials testing the combination of immunotherapy with radiotherapy. J. Immunother. Cancer 4, 51 (2016).

10. Iwai Y, Ishida M, Tanaka Y, Okazaki T, Honjo T, Minato N. Involvement of PD-L1 on tumor cells in the escape from host immune system and tumor immunotherapy by PD-L1 blockade. Proc. Natl Acad. Sci. USA 99(19), 12293-12297 (2002).

11. Lyford-Pike S, Peng S, Young GD et al. Evidence for a role of the PD-1:PD-L1 pathway in immune resistance of HPV-associated head and neck squamous cell carcinoma. Cancer Res. 73(6), 1733-1741 (2013).

12. Partlova S, Boucek J, Kloudova K et al. Distinct patterns of intratumoral immune cell infiltrates in patients with HPV-associated compared to non-virally induced head and neck squamous cell carcinoma. Oncoimmunology 4(1), e965570 (2015).

13. Keytruda ${ }^{\circledR}$ (pembrolizumab) injection, for intravenous use [package insert]. Merck Sharp \& Dohme Corp, Whitehouse Station, NJ, USA (2020).

14. Opdivo (nivolumab) injection, for intravenous use. [package insert]. Bristol-Myers Squibb Company, NJ, USA (2020).

15. EMD Serono and pfizer provide update on Phase III JAVELIN Head and Neck 100 Study [press release]. PR Newswire (13 March 2020).

16. Mehra R, Seiwert TY, Gupta $S$ et al. Efficacy and safety of pembrolizumab in recurrent/metastatic head and neck squamous cell carcinoma: pooled analyses after long-term follow-up in KEYNOTE-012. Br. J. Cancer 119(2), 153-159 (2018). 
- Multicenter, nonrandomized, Phase Ib trial (KEYNOTE-012) assessing pooled efficacy and safety after long-term follow-up in patients included in head and neck squamous cell carcinoma cohorts with recurrent/metastatic disease.

17. Bauml J, Seiwert TY, Pfister DG et al. Pembrolizumab for platinum- and cetuximab-refractory head and neck cancer: results from a single-arm, Phase II study. J. Clin. Oncol. 35(14), 1542-1549 (2017).

- Single-arm Phase II study (KEYNOTE-055) reporting the efficacy and safety of pembrolizumab in patients with platinum- and cetuximab-refractory recurrent/metastatic head and neck squamous cell carcinoma.

18. Cohen EEW, Soulieres D, Le Tourneau C et al. Pembrolizumab versus methotrexate, docetaxel, or cetuximab for recurrent or metastatic head-and-neck squamous cell carcinoma (KEYNOTE-040): a randomised, open-label, phase III study. Lancet 393(10167), 156-167 (2019).

- $\quad$ Randomized, open-label, Phase III trial (KEYNOTE-040) reporting the efficacy and safety of pembrolizumab compared with standard of care (methotrexate, docetaxel or cetuximab) in patients with recurrent/metastatic head and neck squamous cell carcinoma. Treatment with pembrolizumab demonstrated a substantial prolongation of overall survival with a favorable safety profile compared with standard of care.

19. Burtness B, Harrington KJ, Greil R et al. Pembrolizumab alone or with chemotherapy versus cetuximab with chemotherapy for recurrent or metastatic squamous cell carcinoma of the head and neck (KEYNOTE-048): a randomised, open-label, phase III study. Lancet 394(10212), 1915-1928 (2019).

-• Randomized Phase III trial (KEYNOTE-048) reporting efficacy and safety from the final analysis of pembrolizumab monotherapy or pembrolizumab in combination with chemotherapy compared with EXTREME in patients with recurrent/metastatic head and neck squamous cell carcinoma. Pembrolizumab combined with chemotherapy demonstrated superior overall survival in the total population and in patients with a combined positive score $\geq 20$ and $\geq 1$ with a comparable safety profile, whereas pembrolizumab monotherapy demonstrated superior overall survival in patients with a combined positive score $\geq 20$ and $\geq 1$ with a favorable safety profile compared with EXTREME.

20. Powell SF, Gitau MM, Sumey CJ et al. Safety of pembrolizumab with chemoradiation (CRT) in locally advanced squamous cell carcinoma of the head and neck (LA-SCCHN). J. Clin. Oncol. 35(Suppl. 15), 6011-6011 (2017).

- Multisite, single-arm, Phase Ib study (ClinicalTrials.gov: NCT02586207) reporting safety and tolerability of pembrolizumab in combination with standard cisplatin-based chemoradiation in patients with stage III-IVB head and neck squamous cell carcinoma.

21. Powell SF, Gitau M, John Reynolds M et al. Pembrolizumab in combination with chemoradiotherapy (CRT) in human papilloma virus (HPV)-associated head and neck squamous cell carcinoma (HNSCC). [abstr O50]. J. Immunother. Cancer 117 (2018).

- Multisite, single-arm, Phase Ib study (NCT02586207) reporting efficacy of pembrolizumab in combination with standard cisplatin-based chemoradiation in patients with stage III-IVB head and neck squamous cell carcinoma.

22. Eggermont AMM, Blank CU, Mandala M et al. Adjuvant pembrolizumab versus placebo in resected stage III melanoma. NEJM 378(19), 1789-1801 (2018).

23. Edge SB, Byrd DR, Carducci MA. AJCC Cancer Staging Manual. (7th edition.) Springer-Verlag, NY, USA, XV-648 (2010). 
